\title{
CFD modeling of pollution dispersion in building array: Evaluation of turbulent scalar flux modeling in RANS model using LES results
}

\author{
Yoshihide Tominaga $^{\text {a }}$, Ted Stathopoulos ${ }^{b}$ \\ ${ }^{a}$ Department of Architecture and Building Engineering, Niigata Institute of Technology, Kashiwazaki, Niigata, \\ Japan \\ ${ }^{b}$ Centre for Building Studies, Department of Building, Civil and Envir. Engineering, Concordia University, \\ Montreal, Quebec, Canada \\ Corresponding author: Tel./fax: +81257228176. E-mail addresses: tominaga@abe.niit.ac.jp (Y.Tominaga), \\ statho@bcee.concordia.ca (T.Stathopoulos).
}

\begin{abstract}
Modeling of turbulent scalar flux in CFD (Computational Fluid Dynamics) for near-field dispersion around buildings is examined by investigating both velocity and concentration fields obtained by two modeling approaches, i.e., RANS RNG k- $\varepsilon$ and LES. A building array model with a point source located in between the central buildings is adopted as a target configuration. First, the prediction accuracy of LES is confirmed by comparing with the RNGcomputation and the results from an experiment conducted by the authors. LES gives better results than $\mathrm{RNG}$, in terms of time-averaged velocity and concentration distribution in comparison with the wind tunnel experimental results. Next, the eddy viscosity and the eddy diffusivity are determined by LES data using a least square approach as suggested in the dynamic sub-grid scale model. Large differences can be observed between the distributions of the estimated eddy viscosity by using LES data and the eddy diffusivity obtained by RNG, since the eddy diffusivity is not always proportional to the eddy viscosity.
\end{abstract}

\section{Keywords}

Building array, LES, Pollution dispersion, RANS, Turbulent scalar flux

\section{$1 \quad$ Introduction}

Computational Fluid Dynamics (CFD) is widely used to predict wind flow and pollutant dispersion around buildings. Clearly, since the pollutant dispersion around buildings is a complex interaction between the incoming boundary layer, the turbulent flow around buildings and the scalar transport, the prediction accuracy of CFD is strongly influenced by turbulence modeling. The modeling approaches generally have two categories, i.e., RANS (Reynolds-Averaged Navier-Stokes equation model) and LES (Large Eddy Simulation). In order to assess efficiently pollutantdispersion properties around buildings by CFD, it is necessary to clarify the relative performance of the modeling approaches.

In previous studies (Tominaga and Stathopoulos, 2010, 2011), the authors investigated flow and dispersion around a cube and in a simple street canyon by comparing RANSRNG k- $\varepsilon$ (hereafter RNG; Yakhot et al., 1992) and LES in order to examine the performance characteristics of LES in dispersion modeling and to clarify the mechanism of the discrepancy in relation to the RNG computation. These studies confirmed that simple LES modeling gives better results than RNG computation modeling of the distribution of mean concentration in comparison with the wind tunnel experiment. In the case of LES, the horizontal diffusion of concentration is well reproduced and this is mainly due to the reproduction of unsteady concentration fluctuations around buildings. Salim et al. (2011) also reported that LES performed better than RANS (the standard $\mathrm{k}-\varepsilon$ and Reynolds Stress Model) in predicting the concentration distribution in a street canyon. This result was confirmed also in more complex flow fields. Dejoan et al. (2008) assessed and compared RANS and LES for the air flow inside the container's array geometry of the Mock Urban Setting Test (MUST) field experiment. They concluded that LES can predict a higher channeling and thus a higher deflection of the plume, although both modeling approaches are in good qualitative agreement with experimental data. Gousseau et al. (2011a) demonstrated that LES with the dynamic subgrid-scale model shows a better performance than RANS standard k- $\varepsilon$ model in high-resolution CFD simulations of near-field pollutant dispersion in a building group in downtown Montreal. 
The superiority of LES mentioned above is closely related to an intermittent unsteady flow structure, which exists in near-field concentration diffusion around buildings such as building array. Coceal et al. (2006) emphasized that unsteady effects are important, especially in the lower canopy layer where turbulent fluctuations dominate over the mean flow by direct numerical simulations of turbulent flow over regular arrays of urban-like, cubical obstacles. The important difference between unsteady computation like LES and steady-RANS is whether this unsteady nature is reproduced or not. It should be noted that this unsteady nature of flow affects both velocity and concentration fields.

Recently, several papers reported that smaller value of turbulent Schmidt number, $\mathrm{Sc}_{\mathrm{t}}$, which is defined as the ratio of the eddy viscosity to the eddy scalar diffusivity, can provide better results in concentration diffusion in comparison with the case with usual values (0.7-0.9) (e.g. Di Sabatino et al., 2007; Tominaga and Stathopoulos, 2007; Blocken et al., 2008). The improvement with lower $\mathrm{Sc}_{\mathrm{t}}$ can be given that the underestimate of the turbulent scalar diffusion in steady RANS computation is compensated by the low value of $\mathrm{Sc}_{\mathrm{t}}$, as pointed out by the authors (Tominaga and Stathopoulos, 2007). This underestimation is due to the lack of reproduction of unsteadiness in steady-RANS computation. More recently, Chavez et al. (2011) found that variations of $\mathrm{Sc}_{\mathrm{t}}$ have less impact on assessing pollutant dispersion in the presence of adjacent buildings. This supports the above interpretation, because the pollutant transport is well mixed due to the turbulence production by the adjacent buildings. Even though higher order modeling for turbulent scalar flux with an algebraic expression is adopted (e.g. Abe and Suga, 2001; Rossi and Iaccarino, 2009), the problem of the underestimation in turbulent scalar diffusion in steady-RANS still remains. Therefore, the modeling of turbulent scalar flux in RANS model for near-field dispersion around buildings should be examined by investigating both velocity and concentration fields. This will clarify the relative performance of each modeling approach, i.e., steady-RANS, steady-RANS with higher order model, Unsteady-RANS (URANS), LES, DES etc. in prediction techniques for near-field dispersion around buildings.

In the present study, a building array model with a point source located in between the central buildings is adopted as a target configuration. This study is an extension of a previous study (Tominaga and Stathopoulos, 2011), in which basic performance of two modeling approaches, i.e., LES and RNG was evaluated for a simple street canyon model composed of two buildings. In order to investigate the initial stage of the dispersion, special attention is paid to the spatial distribution of the turbulent scalar flux near the source point inside the building canyon. First, the prediction accuracy of LES is confirmed by comparing with a RNG computation and the results from an experiment conducted by the authors. Next, the modeling of turbulent scalar flux in RANS model is examined by comparison with that estimated using the result of LES as a flow and concentration database.

\section{$2 \quad$ Flow and dispersion field}

\subsection{Building array model}

Figure 1 shows the model configuration. In the building array model adopted here, the ambient wind blows perpendicular to the longitudinal side of the buildings. Two parameters of canyon shape, H/W and H/L, are defined as 1.0 and 0.5 , respectively ( $\mathrm{H}$ : building height, $\mathrm{W}$ : distance between buildings and L: building length). In a previous study (Tominaga and Stathopoulos, 2011), only a building canyon formed by two building blocks was considered. However, in the present study, the surrounding building blocks, which have the same shape with a central one, are arranged around the central blocks enclosing the source. Although the building array model is surrounded by the cubic roughness elements $(0.3 \mathrm{H})$ which is smaller than the array building, it should be noted that the upcoming flow developed over the upwind building may differ from an actual extended city. 


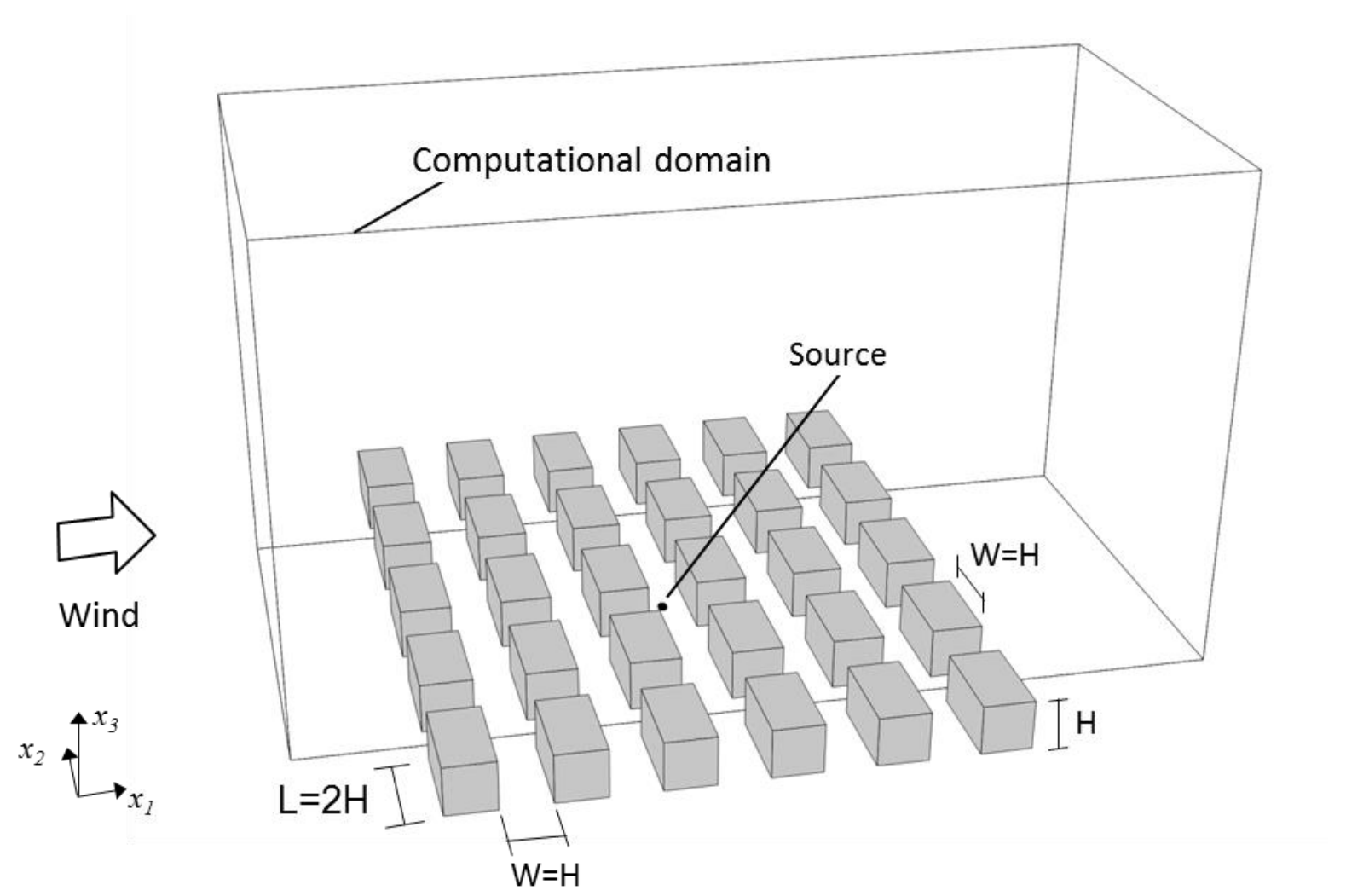

Figure 1. Schematic view of building array model.

\section{Experimental setup}

Experiments were carried out in the boundary layer wind tunnel at Niigata Institute of Technology as a series presented by Tominaga and Stathopoulos (2011). The test section of the boundary layer wind tunnel is $13 \mathrm{~m}$ long, $1.8 \mathrm{~m}$ high and $1.8 \mathrm{~m}$ wide. A combination of spires and surface roughness is used in order to simulate an approach wind profile. The power law exponent of the vertical profile of the inflow velocity is 0.21 . The streamwise turbulence intensity at building height $\mathrm{H}$ is $20 \%$. Passive pollutant was released at a point source at the center of the street bottom. Ethylene $\left(\mathrm{C}_{2} \mathrm{H}_{4}\right)$ was used as a tracer gas. The concentration of emission gas was $1000 \mathrm{ppm}$. The ratio of exit velocity, $\left\langle\mathrm{w}_{\mathrm{S}}\right\rangle$ to $\left\langle\mathrm{u}_{\mathrm{b}}\right\rangle$ was 0.12 , where $\left\langle\mathrm{u}_{\mathrm{b}}\right\rangle=3.8 \mathrm{~m} / \mathrm{s}$ is the upwind mean velocity at building height H. Concentration measurements were performed using a high-speed total Hydro-Carbon Analyzer (Technica; HTHCA-01). Wind velocity was measured by a split fibre probe (Dantec Dynamics; 55R55) and a CTA module, which can discern the three-dimensional components of velocity vector. The time averaging was conducted for a period of $30 \mathrm{sec}$ to obtain statistical values. Repeatability checks were carried out for all measuring points and uncertainty of the time-averaged data was estimated within approximately $\pm 10 \%$ in velocity and $\pm 15 \%$ in concentration.

\section{Computational Setup}

\subsection{Numerical methods}

Numerical methods are fundamentally the same as those in the previous study (Tominaga and Stathopoulos, 2011). The self-developed code used for both RANS and LES computations is based on a finite volume approach for solving flow and concentration equations on structured rectangular grids.

(1) RANS: The RNG model, which shows best agreement with the experiment of the four types of turbulence models in the previous study (Tominaga and Stathopoulos, 2009), was used. The turbulent Schmidt number was set to 0.7 (Tominaga and Stathopoulos, 2007). The QUICK scheme was used for discretizing momentum and concentration equations. Time integration is performed using the implicit Euler scheme, which is first order accurate. Convergence is assumed to be obtained when all the scaled residuals reach $10^{-5}$. 
(2) LES: The standard Smagorinsky model with the empirical constant $\mathrm{C}_{S}=0.12$ was used for the sub-grid scale eddy viscosity model (Smagorisky, 1963). Applicability of the Smagorinsly model with $\mathrm{C}_{S}=0.12$ for a flow around a building model was confirmed in the previous studies (Murakami, 1993; Tominaga et al., 2008a). Near the wall, the length scale was modified by a Van Driest (1956) damping function. The sub-grid scale Schmidt number was set to 0.5 (Antonopoulos-Domis, 1981). A second-order centered difference scheme was adopted for the spatial derivatives. Time integration is performed by using a semi-implicit second-order scheme, while the Adams-Bashforth scheme was explicitly used for the convection term and the CrankNicolson scheme was implicitly used for the diffusion term. The sub-iterations end within a time step when the residual is less than $10^{-5}$, with a maximum of 10 sub-iterations per time step. The time step is set to $\Delta \mathrm{t}$ $=1.0^{-4} \mathrm{~s}$. Before time averaging, the computation was run for 60 non-dimensional time units $\mathrm{t}^{*}\left(=\mathrm{t} \times<\mathrm{u}_{\mathrm{b}}>/ \mathrm{H}\right)$ to remove the influence of an initial condition. Then, the computations were conducted for 200 non-dimensional time units $\mathrm{t}^{*}\left(=\mathrm{t} \times<\mathrm{u}_{\mathrm{b}}>/ \mathrm{H}\right)$, which corresponds to $5 \mathrm{~s}$ in real time scale, to determine the time-averaged values. It was confirmed that the statistical results are almost repeatable with a longer averaging period. Though it is a very important issue whether an averaging time is enough to get a statistically-steady solution in concentration dispersion problem (Schatzmann and Leitl; 2011), in practice, it is subject to the restriction of computational time. In particular, the optimal averaging time in LES is not fully established. In this study, the authors considered that this period is long enough to get statistically-steady values by monitoring evolution of concentration with time at the specific points, although it is smaller than the averaging time in the wind tunnel.

The boundary conditions were set by following the basic guidelines (Tominaga et al., 2008b). The computational domain covered a volume of $18 \mathrm{H}\left(\mathrm{x}_{1}\right) \times 8 \mathrm{H}\left(\mathrm{x}_{2}\right) \times 10 \mathrm{H}\left(\mathrm{x}_{3}\right)$. The computational domain was limited to a few rows of buildings of the experimental geometry. As shown in Figure 1, the computational configuration was composed of three rows of six buildings. It is confirmed in advance that the size of the computational domain does not influence greatly the concentration distribution in the central section near the point source. This computational domain was discretized into $125\left(\mathrm{x}_{1}\right) \times 92\left(\mathrm{x}_{2}\right) \times 42\left(\mathrm{x}_{3}\right)$ grids. 20 cells per building height and 40 cells for building length have been used for the central building blocks. Other cells are gradually coarsened according to the distance from the central building blocks, while the growth ratio of adjacent cells does not exceed 1.3. The point source is modeled as a square shape, which is divided by $2 \times 2$ cells. These conditions were the same in both computations. Only in the RNG computation, it was confirmed that the prediction results did not change significantly with finer grids. Although the grids seem to be rather coarse for LES, the same grids with the RANS computation are intentionally used for the same reason the conventional and simple LES modeling are adopted as mentioned previously (Tominaga and Stathopoulos, 2011).

\subsection{Boundary conditions}

Basic boundary conditions are the same as those used in the previous study (Tominaga and Stathopoulos, 2011) and follow the AIJ guidelines (Tominaga et al., 2008b) in both RNG and LES.Symmetry boundary conditions are prescribed at the top and lateral boundaries and zero gradient condition for all variables is imposed at the outlet plane. The exit face of the stack is defined as a velocity inlet with a uniform velocity profile. Turbulence in the exhaust outlet velocity is not considered. However, the treatment of the boundary conditions for the inlet and the solid wall is different between RNG and LES as described below:

(1) RNG: The vertical distributions of $\left\langle\mathrm{u}_{1}\right\rangle, \mathrm{k}$ and $\varepsilon$ at the inflow boundaries were based on the experiment. The generalized log law was used for the solid boundary (Launder and Spalding, 1974).

(2) LES: A separate LES computation of turbulent boundary layer flow was conducted to generate inflow turbulence. The inflow generating method used here was that proposed by Kataoka and Mizuno (2002). It was confirmed that this method implemented to the computational code used in this study works well for reproducing the velocity and turbulence intensity profiles in generic wind tunnel experiments (Tominaga et al., 2008a; Tominaga and Stathopoulos, 2010). For the boundary condition at the solid walls, a linear or 1/7 power law distribution of instantaneous velocity was assumed (Werner and Wengle, 1991). In this method, a no-slip condition is employed when a first grid point within the viscous sub-layer, or else a 1/7 power-law profile is assumed. 
A flow visualization result with tracer fog releasing from the exit obtained by the wind tunnel experiment is shown in Figure 2. It can be seen that tracer is strongly transferred by clockwise vortex formed in the building canyon. Figures 3 and 4 compare the time-averaged velocities of streamwise $\left(\left\langle u_{1}\right\rangle\right)$ and vertical $\left(\left\langle u_{3}\right\rangle\right)$ components at the center section. The general flow pattern in the building canyon is quite similar with the result for the building canyons without surrounding buildings presented in Tominaga and Stathopoulos (2011). On the distribution of $\left\langle\mathrm{u}_{1}\right\rangle$ as shown in Figure 3, reverse flows indicated as negative values are observed near the bottom of the building canyons. The location of the negative peak in LES is closer to the bottom than that in RNG. Furthermore, on the distribution of $\left\langle u_{3}\right\rangle$ as shown in Figure 4, LES provides the stronger downwash flow in front of the leeward building than RNG. That is, the recirculation flow predicted by LES is a little stronger than that in RNG in the building canyon. Figure 5 compares the vertical distributions of streamwise velocity above the source point. The velocity value near the bottom obtained by LES is closer to the experimental values than that by RNG, though the general agreement between two computations and the experiment is good.

Figure 6 shows the time-averaged concentrations at the center section. Concentrations are nondimensionalized by the reference concentration $\left\langle c_{0}\right\rangle$ given by:

$$
<c_{0}>=\frac{Q_{e}}{H^{2}<u_{b}>}
$$

where $\mathrm{Q}_{\mathrm{e}}$ is the pollutant exhaust rate.

By comparing with the experimental results without the surrounding buildings (Tominaga and Stathopoulos, 2011), the distribution pattern is similar, but computed concentration values are slightly higher near the source. This difference is caused by the influence of the surroundings, by which the advection of concentration becomes small and its turbulent diffusion becomes large in the building canyon. In the computational results, the concentration behind the upwind building in RANS is higher than that in LES. This means the concentration transport along the upwind direction by advection is dominant in the results obtained by RNG. Therefore, the concentration in the region upwind from the source point in RNG is larger than that in LES and the experiment. This larger concentration is closely related to the turbulent diffusion that is smaller in RNG as will be discussed later in this paper. The comparison of vertical distribution of concentration is shown in Figure 7 . The agreement with the experiment in LES is better than that in RANS. Notably, the upper region at the plotting line at $\mathrm{x}_{1} / \mathrm{H}=-0.4$, the predicted concentration by LES, which is much lower than that in RNG, is much close to the experimental results.

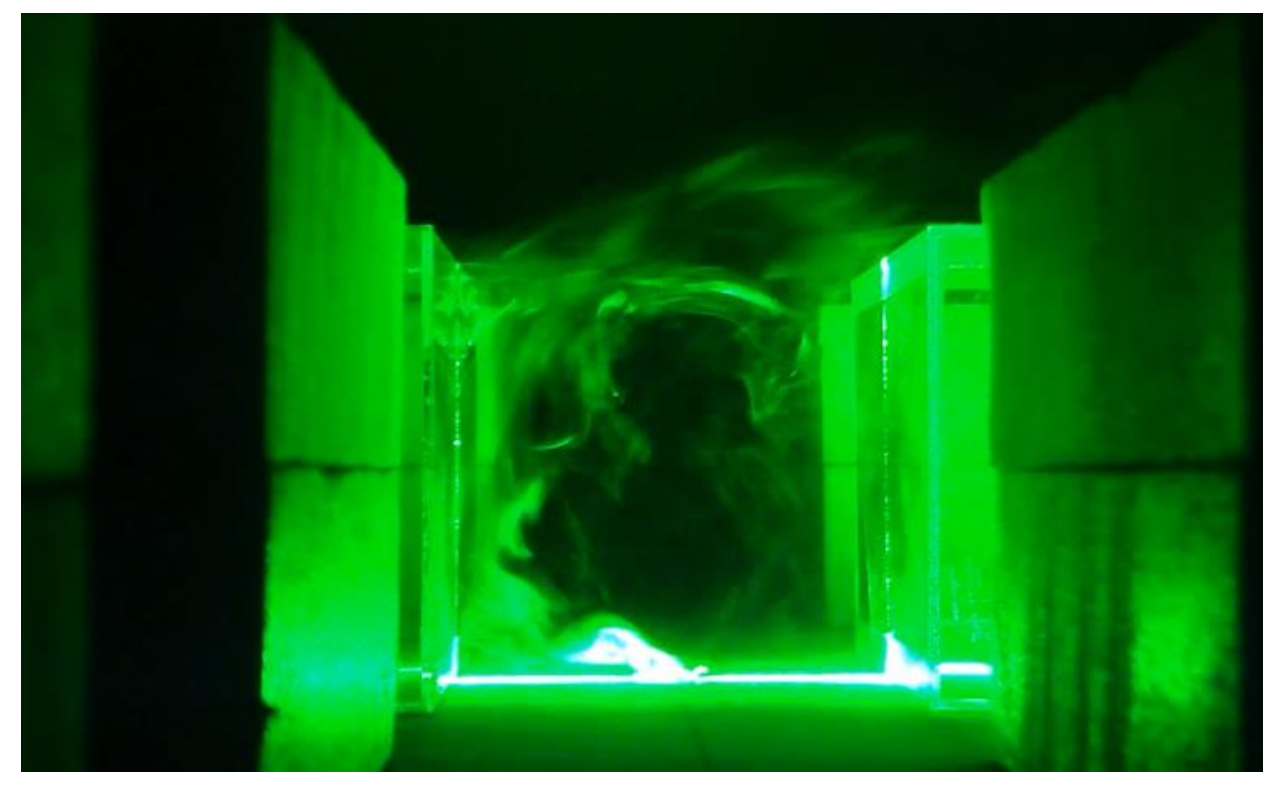

Figure 2. Flow visualization with tracer fog releasing from exit obtained by wind tunnel experiment. 
(a)

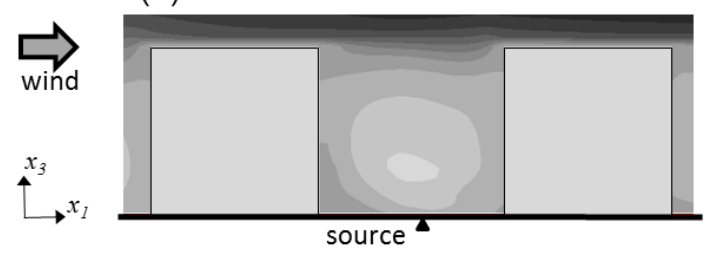

(b)

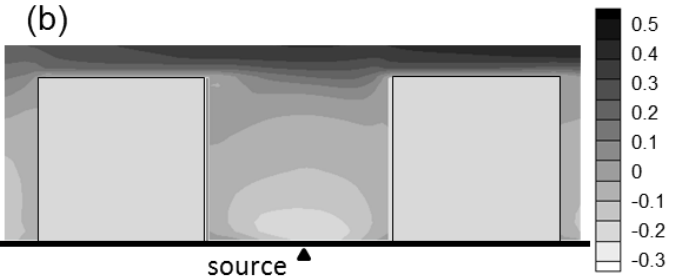

Figure 3. Time-averaged velocity in streamwise component $\left(\left\langle\mathrm{u}_{1}\right\rangle\right)$ inside a building canyon at vertical center section: (a) RNG, (b) LES.

(a)

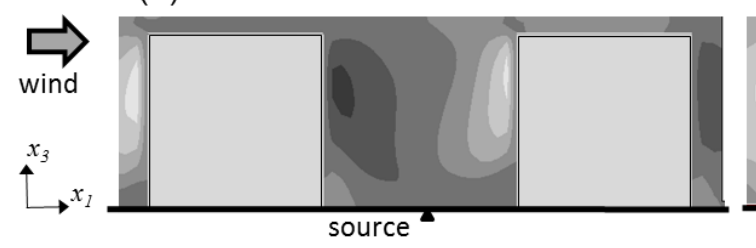

(b)

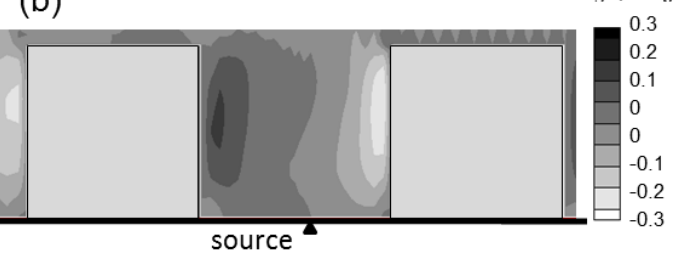

Figure 4. Time-averaged velocity in vertical component $\left(\left\langle\mathrm{u}_{3}\right\rangle\right)$ inside a building canyon at vertical center section: (a) RNG, (b) LES.

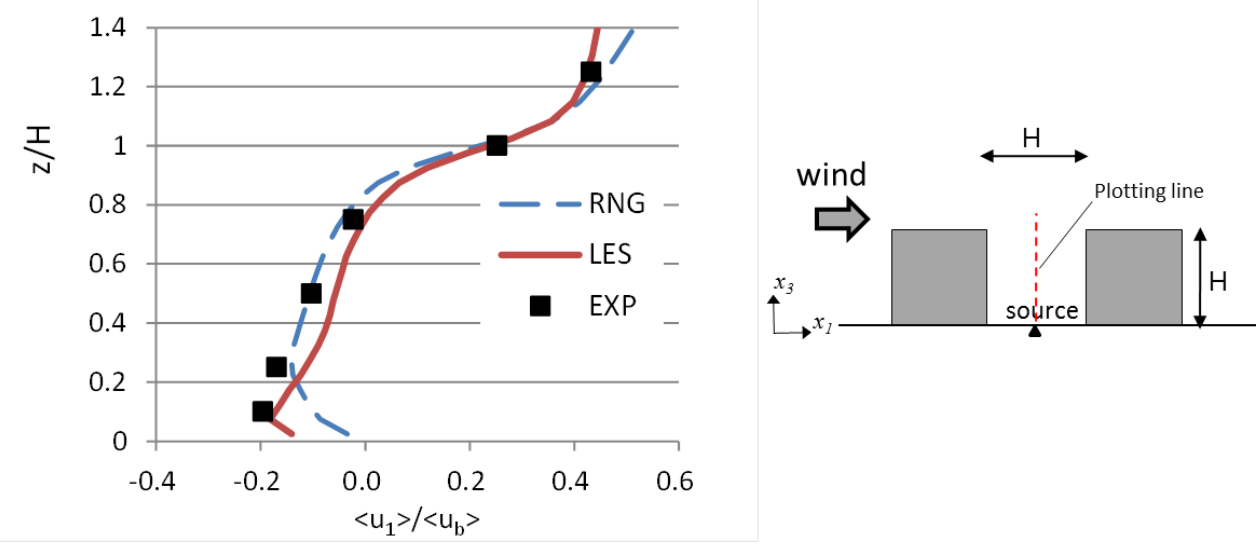

Figure 5. Comparison of time-averaged streamwise velocity above a source point.

(a)

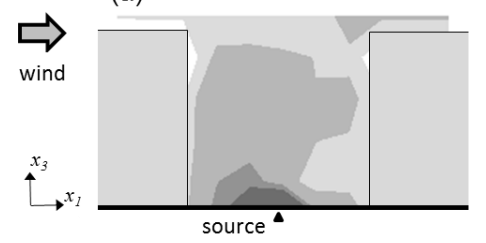

(b)

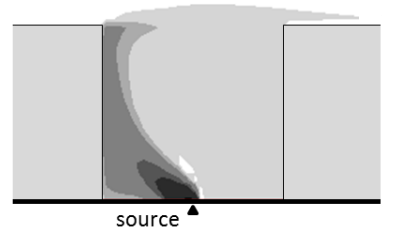

(c)

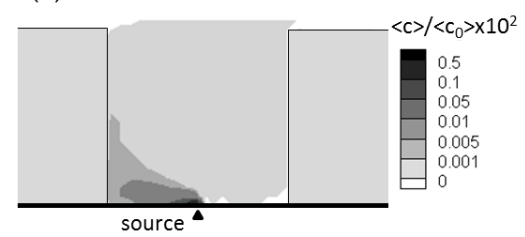

Figure 6. Time-averaged concentration inside a building canyon at vertical center section: (a) experiment, (b) RNG, (c) LES. 

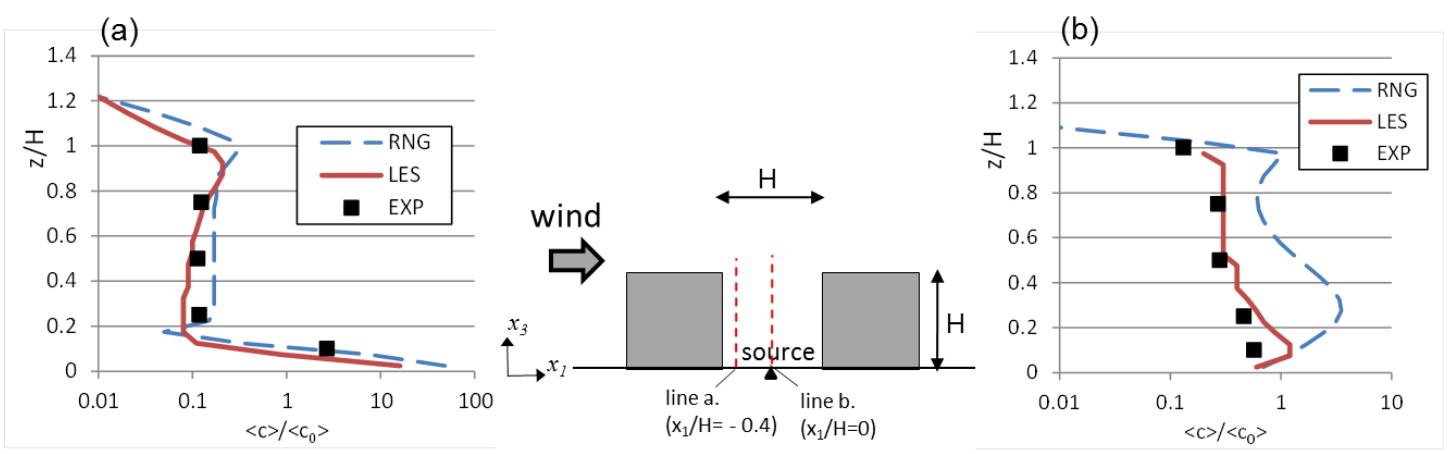

Figure 7. Comparison of vertical distributions of time-averaged concentration: (a) $\mathrm{x}_{1} / \mathrm{H}=-0.4$, (b) $\mathrm{x}_{1} / \mathrm{H}=0$.

\section{Evaluation of eddy viscosity and eddy diffusivity using LES results}

In the k- $\varepsilon$ type RANS models, the unknown Reynolds stresses are obtained from the linear relationship between the Reynolds stress $\left\langle u_{i}{ }^{\prime} u_{j}{ }^{\prime}\right\rangle$ and the mean strain rate $S_{i j}$ :

$$
\begin{aligned}
& <u_{i}{ }^{\prime} u_{j}{ }^{\prime}>=-2 v_{t} S_{i j}+\frac{2}{3} k \delta_{i j} \\
& S_{i j}=\frac{1}{2}\left(\frac{\partial<u_{i}>}{\partial x_{j}}+\frac{\partial<u_{j}>}{\partial x_{i}}\right)
\end{aligned}
$$

On the other hand, the Reynolds stresses, at least in grid scales, are calculated directly in LES. When the Reynolds stresses and the mean strain rate in Eq. (1) are given by LES data, the eddy viscosity $v_{t}$ has six independent tensor elements. Here, the eddy viscosity $v_{t}$ is determined using a least square approach as suggested in the dynamic sub-grid scale model (Lilly, 1992):

$$
v_{t}=-\frac{<u_{i}{ }^{\prime} u_{j}{ }^{\prime}>S_{i j}}{S_{i j}{ }^{2}}
$$

Figure 8 illustrates the distribution of the eddy viscosity obtained by RNG model and those estimated by using LES data. It should be noted that the contours obtained by LES data are not smooth because the momentum and scalar transport are actually three dimensional and anisotropic. However, this approach is useful in order to estimate the expected value of the eddy viscosity and the eddy diffusivity. Although the distribution patterns are somewhat similar, the large peaks, which are not observed in RNG, appear above the buildings and inside the building canyon in the values obtained by LES. The value inside the building canyon in RNG is much smaller than that in LES. This underestimation in RNG is mainly caused by the absence of time-dependent fluctuation inside the building canyon (Tominaga and Stathopoulos, 2010, 2011).

The distribution of concentration fluxes represents the essentials of concentration transport, because it can provide very important information for investigating the validity of a model used for concentration transport. Scalar transport of concentration consists of convective and turbulent diffusion effects, which are expressed by convection as mean scalar fluxes $\left\langle\mathrm{u}_{\mathrm{i}}\right\rangle\langle\mathrm{c}\rangle$ and turbulent diffusion fluxes $\left\langle\mathrm{u}_{\mathrm{i}}{ }^{\prime} \mathrm{c}\right.$ ' $\rangle$, respectively. The convective fluxes can be estimated by using mean velocities $\left\langle\mathrm{u}_{\mathrm{i}}\right\rangle$ and mean concentration $\langle\mathrm{c}\rangle$. The turbulent diffusion fluxes are calculated directly in LES. On the other hand, in the k- $\varepsilon$ type RANS models, the scalar flux is estimated using the gradient diffusion hypothesis as shown in Eq. (4) with the eddy diffusivity $\mathrm{D}_{\mathrm{t}}$ usually expressed by the eddy viscosity and the turbulent Schmidt number as per Eq. (5):

$$
\begin{aligned}
& <u_{i}{ }^{\prime} c^{\prime}>=-D_{t} \frac{\partial<c>}{\partial x_{i}} \\
& D_{t}=\frac{v_{t}}{S c_{t}}
\end{aligned}
$$

Figure 9 compares the streamwise component $\left(\left\langle\mathrm{u}_{1}{ }^{\prime} \mathrm{c}^{\prime}\right\rangle\right)$ and the vertical components $\left(\left\langle\mathrm{u}_{3}{ }^{\prime} \mathrm{c}^{\prime}\right\rangle\right)$ of turbulent diffusion fluxes on the vertical sections. In the distribution of $\left\langle\mathrm{u}_{1}{ }^{\prime} \mathrm{c}^{\prime}\right\rangle$, although the distributions are similar in both computations, the larger value along with the plume from the source in RNG extends to the upper region of the building canyon more than in the LES case. RNG also shows larger negative values upwind of the source in 
comparison with LES. On the other hand, in the distribution of $\left\langle\mathrm{u}_{3}{ }^{\prime} \mathrm{c}^{\prime}\right\rangle$, a large difference between the two models is observed in the upwind region from the source. The larger value of the flux between the upwind building and the source is observed in LES than in RNG. Consequently, LES shows a much larger contribution of turbulent diffusion fluxes than RNG in the region upwind from the source. The large difference between the modeled turbulent fluxes suggests that the accuracy of the turbulent diffusion modeling is very important in predicting the mean concentration distribution. This remark was also pointed out by Gousseau et al. (2011b).

By applying the same approach used for estimating the eddy viscosity $v_{t}$ into Eq. (4), the estimated eddy diffusivity $D_{t}$ can be obtained by using LES data. Figure 10 illustrates the distribution of the eddy diffusivity $D_{t}$ obtained by the RNG model and also calculated by using LES data. Understandably, the distribution of the values from RNG has a complete similarity to that of eddy viscosity $v_{t}$. However, differences can be observed between the distributions of the estimated eddy viscosity as indicated in Figure 8 and the eddy diffusivity from LES data, since the eddy diffusivity is not always proportional to the eddy viscosity in this case. This failure on the gradient diffusion hypothesis is closely related to the fact that the turbulent Schmidt number $\mathrm{Sc}_{t}$ in RANS model is often problematic for scalar transport in complex flow fields such as flow around buildings (Tominaga and Stathopoulos, 2007).

By using Eq. (5) with the estimated values of the eddy viscosity $v_{t}$ and the eddy diffusivity $\mathrm{D}_{\mathrm{t}}$, an estimated turbulent $\mathrm{Schmidt}$ number $\mathrm{Sc}_{\mathrm{t}}$ can be obtained. Figure 11 illustrates the distribution of $\mathrm{Sc}_{\mathrm{t}}$ estimated by LES data. Although some discontinuity can be observed in the contour due to the limitation of isotropic assumption mentioned previously, $\mathrm{Sc}_{\mathrm{t}}$ values vary within the approximate range of 0.2 to 2.0. In most regions of the building canyon, the estimated values of $\mathrm{Sc}_{\mathrm{t}}$ are much smaller than 1.0. This result is consistent with previous findings in similar dispersion studies, in which a smaller value of $\mathrm{Sc}_{\mathrm{t}}$ often provides better results than the usual value - say 0.7 (e.g. Di Sabatino et al, 2007; Blocken et al., 2008; Gousseau et al, 2011). Meanwhile, the large value of $\mathrm{Sc}_{\mathrm{t}}$ is observed in the region upwind from the source. The large variety of $\mathrm{Sc}_{\mathrm{t}}$ presented here suggests that anisotropy should be considered for predicting the turbulent scalar flux in complex dispersion fields adequately (Rossi and Iaccarino, 2009). However, it is difficult to generalize these high order modelings for highly complex threedimensional flows such as that of near-field dispersion around buildings, because many numerical parameters to be optimized appear in such models.

(a)

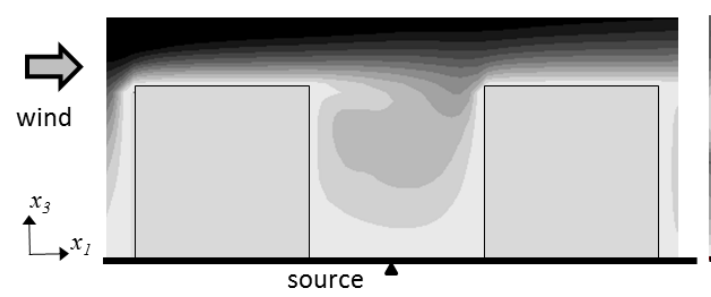

(b)

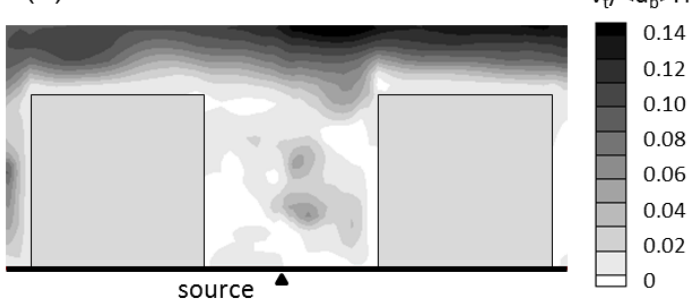

Figure 8. Eddy viscosity $v_{t}$ :(a) values obtained by RNG model, (b) values estimated by using LES data.

(a)

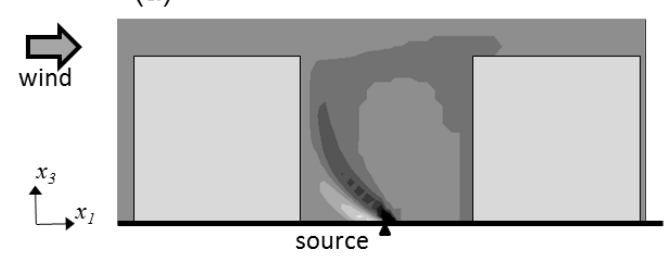

(c)

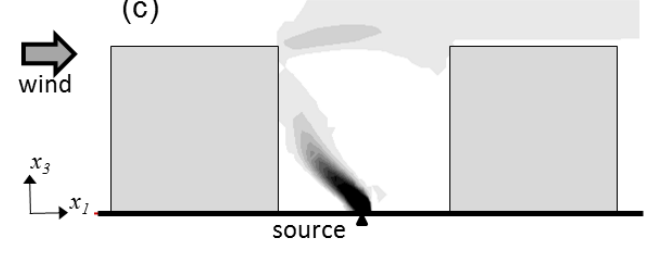

(b)

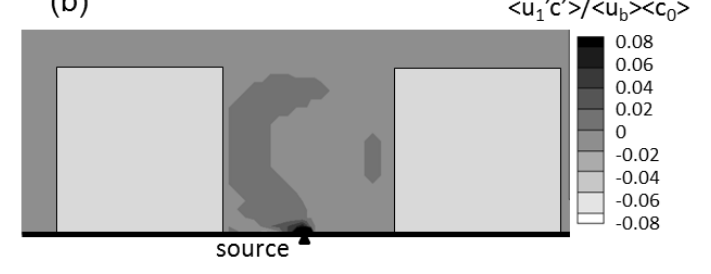

(d)

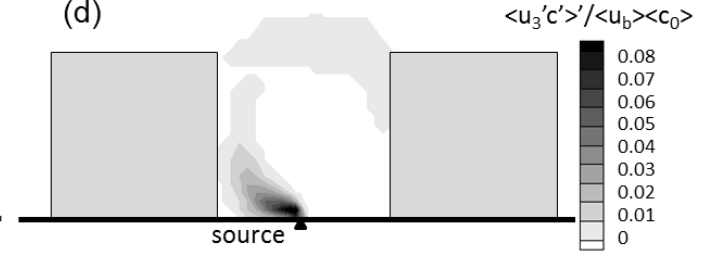

Figure 9. Turbulent scalar fluxes of streamwise (upper; $\left\langle u_{1}{ }^{\prime} c^{\prime}\right\rangle$ ) and vertical (lower; $<u_{3}$ 'c'>) components: (a, c) RNG, (b, d) LES. 
(a)

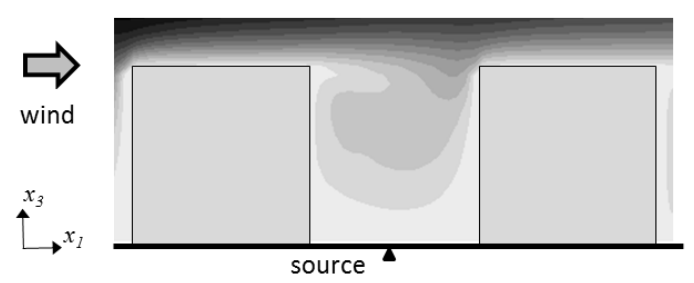

(b)

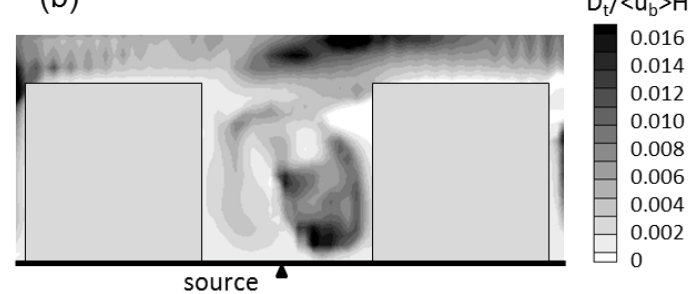

Figure 10. Eddy diffusivity $D_{t}$ :(a) values obtained by RNG model, (b) values estimated by using LES data.

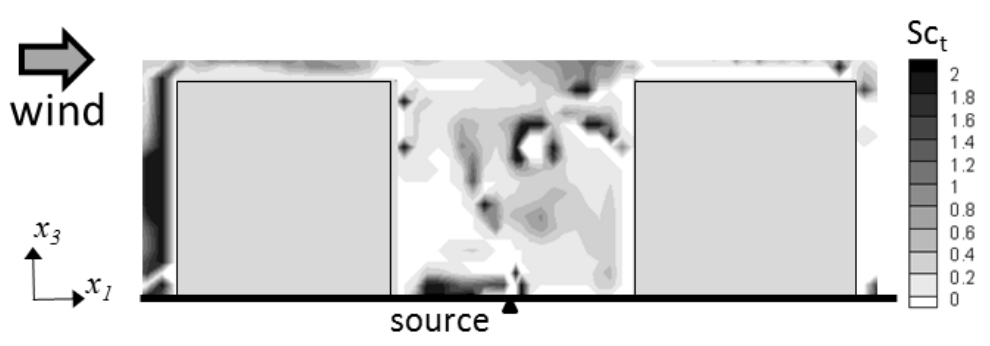

Figure 11. Estimated turbulent Schmidt number $\mathrm{Sc}_{\mathrm{t}}$ by LES data.

\subsection{Prediction accuracy on flow field to mean concentration field using LES results}

Clearly, since the modeling of turbulent scalar diffusion flux (Eq. (4)) contains the eddy viscosity $v_{t}$, it is strongly affected by the prediction accuracy of turbulent flow field. If predicted values of $v_{\mathrm{t}}$ in the model are significantly different from the actual property in real phenomena, $\mathrm{Sc}_{\mathrm{t}}$. number can be used for compensation purposes. In this section, in order to clarify this effect, a numerical experiment is carried out. In this experiment, the Reynolds averaged transport equations shown as Eq. (6) are solved using the time-averaged flow field obtained by LES. The distribution of $v_{\mathrm{t}}$ estimated by the result from LES, shown previously in Figure 8, is also used for the computation. Here, $\mathrm{Sc}_{\mathrm{t}}$ is set at 0.7 as well as the RNG case.

$\frac{\partial<c>}{\partial t}+<u_{i}>\frac{\partial<c>}{\partial x_{i}}=-\frac{\partial}{\partial x_{i}}\left(\frac{v_{t}}{S c_{t}} \frac{\partial<c>}{\partial x_{i}}\right)$

The averaged concentration distribution obtained by the numerical experiment is shown in Figure 12. Clearly, the concentration transport to the upwind region near the ground becomes large and results come rather close to the experimental data in comparison with the RNG results shown in Figure 6. This supports the superiority of flow field prediction in LES, which is mainly caused by the reproduction of the unsteady nature of the flow around buildings, which has much influence on the predicted result in the concentration field. In other words, by reproducing the unsteady nature in the flow field, the influence of the modeling can be small in turbulent diffusion flux including $\mathrm{Sc}_{\mathrm{t}}$. Therefore, the applicability of unsteady-RANS (URANS) should be investigated further as a possible route to solve these problems (e.g. Iaccarino et al., 2003).

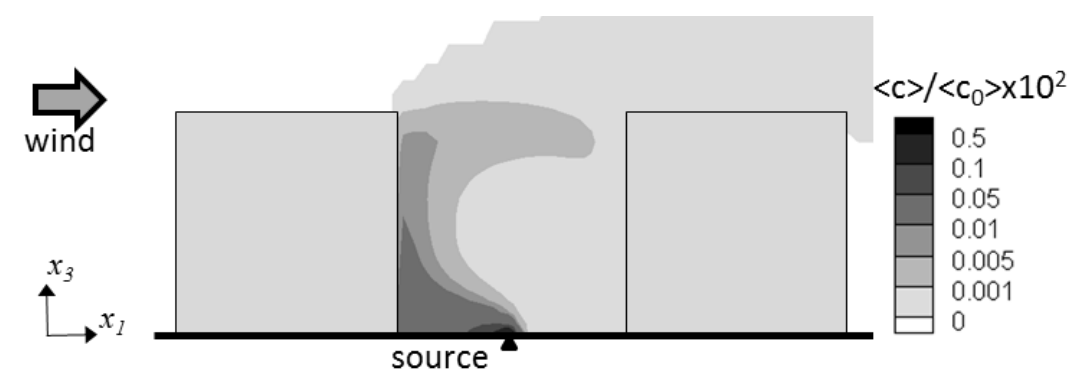

Figure 12. Time-averaged concentration obtained by Reynolds averaged transport equation for $\langle\mathrm{c}\rangle$ using timeaveraged flow filed obtained by LES. 
This work investigates the spatial distribution of the turbulent scalar flux inside the building array with a point source by comparing the numerical results obtained by LES and RNG computations. Basic performance of each modeling approach for this configuration is confirmed as the same as that for the simple street canyon model presented in the previous study (Tominaga and Stathopoulos; 2011). That is, RNG underestimates turbulence diffusion in the building canyon when compared with LES due to the difference in the representation of turbulent scalar flux.

As a unique feature of this study, the eddy viscosity and the eddy diffusivity are determined using a least square approach as suggested in the dynamic sub-grid scale model. Large differences can be observed between the distributions of the estimated eddy viscosity and the eddy diffusivity, since the eddy diffusivity is not always proportional to the eddy viscosity. The turbulent Schmidt number is also estimated by LES data and values vary in the 0.2 to 2.0 approximate range. The large variety of $\mathrm{Sc}_{\mathrm{t}}$ suggests that anisotropy should be considered for precisely predicting the turbulent scalar flux in complex dispersion fields such as those in building arrays.

Furthermore, a numerical experiment, in which the Reynolds-averaged transport equations are solved using the time-averaged flow field obtained by LES, has been carried out. The superiority of flow field in LES has been shown to have much influence on the concentration diffusion field. Therefore, the applicability of unsteadyRANS (URANS) should be investigated further as one possibility to solve these problems in a future study.

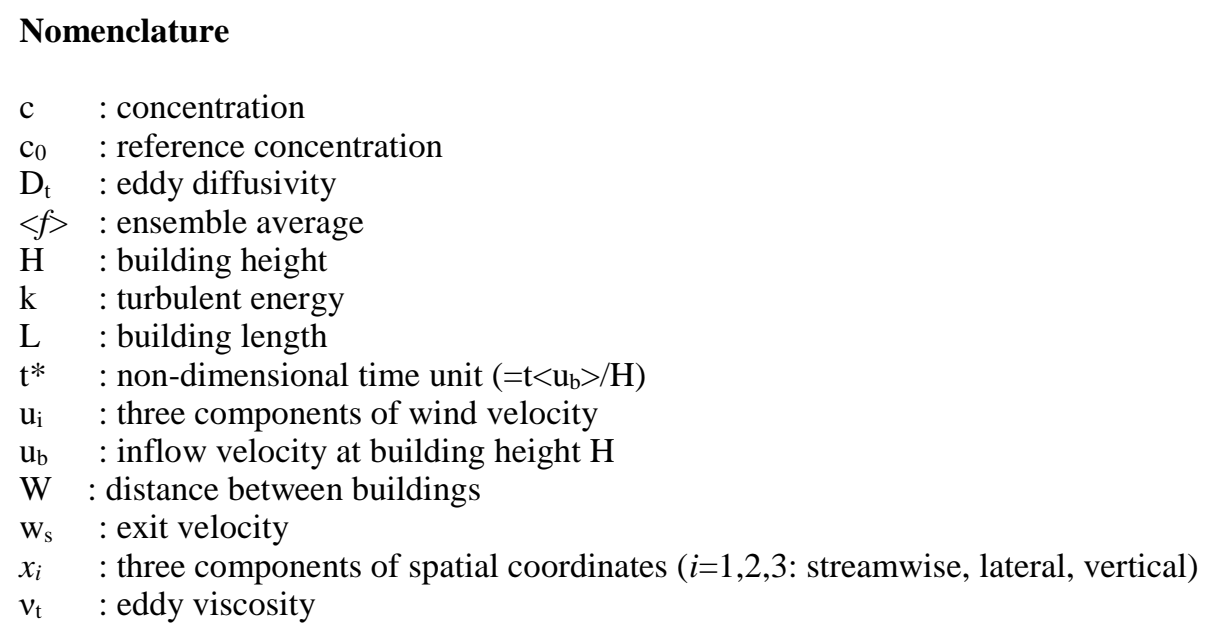

\section{Acknowledgements}

This work was financially supported by the Grants-in-Aid for Scientific Research in Japan (No. 21560622) and the Obayashi Foundation. This paper was partially provided during the period the first author worked as a visiting scientist in the Department of Building, Civil and Environmental Engineering of Concordia University, Montreal, Canada in 2011. The authors would like to express their gratitude to FY 2011 Researcher Exchange Program between JSPS (Japan Society for the Promotion of Science) and NSERC (Natural Sciences and Engineering Research Council of Canada) for supporting this visit.

\section{References}

Abe, K., Suga, K., 2001. Toward the development of a Reynolds-averaged algebraic turbulent scalar flux model. International Journal of Heat and Fluid Flow 22, 19-29.

Antonopoulos-Domis, M., 1981. Large eddy simulation of a passive scalar in isotropic turbulence, Journal of Fluid Mechanics 104, 55-79.

Blocken, B., Stathopoulos, T., Saathoff, P. and Wang, X., 2008. Numerical evaluation of pollutant dispersion in the built environment: comparisons between models and experiments. Journal of Wind Engineering and Industrial Aerodynamics 96, 1817-1831.

Chavez, M., Hajra, B., Stathopoulos, T., Bahloul, A., 2011. Near-field pollutant dispersion in the built environment by CFD and wind tunnel simulations. Journal of Wind Engineering and Industrial Aerodynamics 99, 330-339.

Coceal, O., Thomas, T. G., Castro, I. P., Belcher, S. E., 2006. Mean flow and turbulence statistics over groups of urban-like cubical obstacles. Boundary-Layer Meteorology 121, 491-519. 
Dejoan, A., Santiago, J., Martilli, A., Martin, F., Pinelli, A. 2008. Comparison between large-eddy simulation and Reynolds-averaged Navier-Stokes computations for the MUST field experiment. Part II: Effects of incident wind angle deviation on the mean flow and plume dispersion. Boundary-Layer Meteorology 135, 133-150.

Di Sabatino S., Buccolieri, R., Pulvirenti, B., Britter, R., 2007. Simulations of pollutant dispersion within idealised urban-type geometries using CFD and integral models. Atmospheric Environment 41, 8316-8329.

Gousseau, P., Blocken, B, Stathopoulos, T, van Heijst, G.J.F., 2011a. CFD simulation of near-field pollutant dispersion on a high-resolution grid: a case study by LES and RANS for a building group in downtown Montreal. Atmospheric Environment 45, 428-438.

Gousseau, P., Blocken, B., van Heijst G.J.F., 2011b. CFD simulation of pollutant dispersion around isolated buildings: On the role of convective and turbulent mass fluxes in the prediction accuracy. Journal of Hazardous Materials 194, 422-434.

Iaccarino, G., Ooi, A., Durbin, P.A., Behnia, M., 2003. Reynolds Averaged Simulations of Unsteady Separated Flow, International Journal of Heat and Fluid Flow 24, 147-156.

Kataoka, H., Mizuno, M., 2002. Numerical flow computation around aeroelastic 3D cylinder using inflow turbulence, Wind and Structures 5, 379-292.

Launder, B.E., Spalding, D.B., 1974. The numerical computation of turbulent flows. Computer Methods in Applied Mechanics and Engineering 3, 269-289.

Lilly, D.K., 1992, A proposed modification of the Germano subgrid-scale closure method. Physics of Fluids A4, 633-635.

Moonen, P., Dorer, V., Carmeliet, J., 2011. Evaluation of the ventilation potential of courtyards and urban street canyons using RANS and LES. Journal of Wind Engineering and Industrial Aerodynamics 99, 414-423.

Murakami, S., 1993. Comparison of various turbulence models applied to a bluff body. Journal of Wind Engineering and Industrial Aerodynamics 46-47, 21-36.

Rossi, R, Iaccarino, G., 2009. Numerical simulation of scalar dispersion downstream of a square obstacle using gradient-transport type models. Atmospheric Environment 43, 2518-2531.

Salim S. M., Buccolieri, R., Chan, A., Di Sabatino, S., 2011. Numerical simulations of atmospheric pollutant dispersion in an urban street canyon: Comparison between RANS and LES. Journal of Wind Engineering and Industrial Aerodynamics 99, 103-113.

Schatzmann, M., Leitl, B, 2011. Issues with validation of urban flow and dispersion CFD models, Journal of Wind Engineering and Industrial Aerodynamics 99, 169-186.

Smagorinsky, J., 1963. General circulation experiments with the primitive equations I. The basic experiment. Monthly Weather Review 91, 99-164.

Tominaga, Y. and Stathopoulos, T., 2007. Turbulent Schmidt numbers for CFD analysis with various types of flowfield. Atmospheric Environment 41, 8091-8099.

Tominaga, Y., Mochida, A., Murakami, S., Sawaki, S., 2008a. Comparison of various revised k- $\varepsilon$ models and LES applied to flow around a high-rise building model with 1:1:2 shape placed within the surface boundary layer. Journal of Wind Engineering and Industrial Aerodynamics 96, 389-411.

Tominaga, Y., Mochida, A., Yoshie, R., Kataoka, H., Nozu, T., Yoshikawa, M., Shirasawa, T., 2008b. AIJ guidelines for practical applications of CFD to pedestrian wind environment around buildings, Journal of Wind Engineering and Industrial Aerodynamics 96, 1749-1761.

Tominaga, Y., Stathopoulos, T., 2009. Numerical simulation of dispersion around an isolated cubic building : Comparison of various types of k- $\varepsilon$ models. Atmospheric Environment 43, 3200-3210.

Tominaga, Y., Stathopoulos, T., 2010. Numerical simulation of dispersion around an isolated cubic building: Model evaluation of RANS and LES, Building and Environment 45, 2231-2239.

Tominaga, Y., Stathopoulos, T., 2011. CFD Modeling of Pollution Dispersion in a Street Canyon: Comparison between LES and RANS. Journal of Wind Engineering \& Industrial Aerodynamics 99, 340-348.

Van Driest, E. R., 1956. On turbulent flow near a wall, Journal of Aeronautical Science 23, 1007-1011.

Werner, H., Wengle, H., 1991. Large eddy simulation of turbulent flow over and around a cube in plane channel. In : Proceedings of 8th Symposium on Turbulent Shear Flows, 155-168.

Yakhot, V., Orszag, S. A., Thangam, S., Gatski, T. B., Speziale, C. G., 1992. Development of turbulence models for shear flows by a double expansion technique. Phys. Fluids A 4, 1510-1520. 